\title{
Summary of Application of Magnetic Coupling Resonance Technology in Power Inspection UAV
}

\author{
Ji Jiang ${ }^{1}$, Xuanyi Liu ${ }^{1}$ and Xiaoming Zha ${ }^{1}$ \\ ${ }^{1}$ School of Electrical Engineering, Wuhan University, China
}

\begin{abstract}
With the improvement of technology, technology began to gradually applied to every field, electric field inspection also began to gradually enable unmanned aerial vehicle (uav) inspection instead of manual inspection. Articles in the domestic and foreign research literature of unmanned aerial vehicle (uav) magnetic coupling resonance charging basis, this paper introduces the unmanned aerial vehicle (uav) magnetic coupling resonance charging technology development and application of the status quo, points out the problems in the current study of, offers a new way for the further research in this field.
\end{abstract}

Keywords: Unmanned aerial vehicles (UAVS); Wireless charging. Magnetic coupling resonance; Application research on.

\section{Preface}

Power inspection is one of the most important aspects of the power industry. Without a good inspection mechanism, it is impossible to guarantee the complete and orderly operation of a power system. At present, most of the power inspection methods adopted in China still remain in the original mode of manual inspection, and the manual method has its advantages, that is, the sufficient experience of the inspection workers and the good emergency mechanism make the failure can be solved as quickly as possible. However, there are still some disadvantages, that is, more time is consumed, and other special terrains, including forests and deserts, have certain risks. Therefore, the use of modern inspection methods instead of manual methods is particularly demanding, and drones are a popular choice for the moment. Compared with labor, drones have the advantages of high inspection efficiency and ignoring terrain obstacles, but they also have their own disadvantages, which is the problem of battery life.

Therefore, it is necessary to introduce wireless charging technology into the drone. How to enable the drone to continue the inspection process independently is a hot issue. At present, there are three main forms of radio energy transmission technology: inductive, resonant and microwave radio transmission. ${ }^{[1]}$ Among them, the inductive transmission distance is too short, and the microwave radio can transmit too long. Therefore, the current magnetic coupling resonant charging method is the mainstream of the UAV charging method research.

\section{Magnetic coupling principle and composition \\ resonance}

In 2007, Marin Soljiacic, a professor of physics at the Massachusetts Institute of Technology, published a paper on resonant radio energy transmission technology in Science. Since then, resonant radio energy transmission technology has begun to enter people's field of vision and has caused a series of research booms. The main content is two coupled coils with the same resonant frequency, which generate resonance through the electromagnetic field, thereby transmitting energy, while other components with different frequencies are basically unaffected, that is, a resonant wireless energy transmission system with a time-varying electromagnetic field as a coupling medium. ${ }^{[2]}$

In 2007, the MIT research team experimentally verified the theory by applying two circular solenoids as energy-transmitting resonators. The specific parameters are: solenoid number $n=5.25$, diameter $d=60 \mathrm{~cm}$, height $\mathrm{h}=20 \mathrm{~cm}$, wire diameter $\mathrm{a}=6 \mathrm{~mm}$, system resonance frequency $\mathrm{f}=9.9 \mathrm{MHz}$, theoretical value of quality factor $\mathrm{Q}=2500$, actual measured value $\mathrm{Q}^{\prime}=950$. In the test, the team used a single-twisted copper wire loop as the drive circuit. The two solenoids were coupled to each other and connected to a $60 \mathrm{~W}$ bulb. Finally, the experiment successfully illuminates the bulb with a distance of $2.16 \mathrm{~m}$, and the energy transmission efficiency reaches $40 \%$. When the experimental team adjusted the distance to $1 \mathrm{~m}$, the transmission efficiency was close to $90 \%$. The overall experiment verified the feasibility of the theory. 


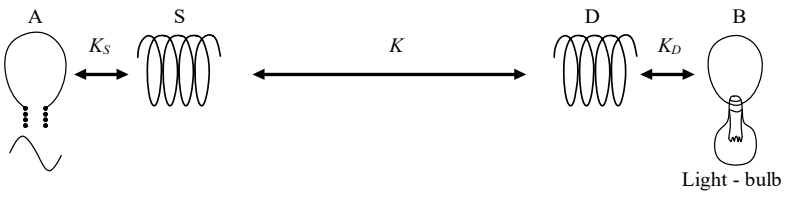

Figure 1. Basic structure of a magnetically coupled resonant radio energy transmission system.

\section{Foreign research progress}

Foreign research on magnetic coupling resonant charging has been in the blowout state in recent years.

In 2009, Japan's Takehiro et al. increased the coil radius from $300 \mathrm{~mm}$ to $150 \mathrm{~mm}$ by increasing the coupling capacitance, achieving wireless transmission of $100 \mathrm{~W}$ of power within $200 \mathrm{~m}$, and ensuring transmission efficiency of over $95 \%{ }^{[3]}$. In 2010 , TC Beh et al. studied the application of magnetic coupling resonant charging method in electric vehicles, and proposed a method to further improve transmission efficiency, that is, using impedance matching circuit to adjust the resonant frequency of the resonator ${ }^{[4]}$. In 2011, the Korean team achieved the best matching of the system impedance by adjusting the distance between the two resonant coils. In the same year, Seung-Hwan Lee et al. proposed a magnetically coupled resonant wireless transmission with a surface helical coil on the surface. The equivalent model of the system ${ }^{[5]}$. In 2012, A4WP, a wireless charging alliance based on resonant wireless transmission technology, was formally established. At present, more than ten companies such as Philips, Samsung and Microsoft have joined the alliance. In 2014, the National Academy of Science and Technology of Korea proposed a technology to charge multiple smartphones within 5 meters.

\section{Domestic research progress}

China's research on magnetically coupled resonance technology started late, but it has also achieved certain results.

Among them, Zhu Chunbo's research group of Harbin Institute of Technology in 2008 was the first research group of magnetic coupling resonance technology in China. They proposed three methods to increase the transmission distance, namely adding the booster, increasing the power supply voltage and adopting multi-stranded wires in parallel coils, and experimentally verified that these three methods can effectively improve the energy transmission distance ${ }^{[6]}$. In 2009, Zhang Bo's research group of South China University of Technology proposed an optimization method for wireless energy transmission system, which is to construct a small-sized single-turn spiral coil resonance system model. From the circuit point of view, analyze the load coil, coil size, distance and other factors and wireless energy transmission. The relationship between the systems, and the corresponding expression [7]. In 2011, Huang Xueliang's research group of Southeast University conducted a test to investigate the transmission efficiency under extreme conditions, and pointed out that by increasing the resonant frequency, coil radius, coil turns, reducing high-frequency resistance loss, and reducing the inherent attenuation frequency, the transmission distance can be effectively improved. ${ }^{[8]}$

In addition, Chongqing University has developed a radio energy transmission prototype to study the distributed radio energy transmission mode ${ }^{\text {[9]; Tianjin }}$ University of Technology has established a mathematical model to analyze the frequency splitting characteristics of the system ${ }^{[10]}$.

\section{Magnetic coupling resonant charging method applied to power inspection drones}

The development of magnetically coupled resonant charging methods is changing with each passing day. As technology becomes more mature, the technology will be more popularized and applied. However, in the application process, there are inevitably some technical problems that need to be solved. In the application of power inspection drones, there are mainly several problems:

(1) When the UAV performs magnetic coupling resonance wireless charging, it is not completely statically parked on the charging platform, and is in a semi-suspended state, so the charging distance, the load size, and the coupling capacitance are constantly changing. Under constant frequency conditions, a stable resonance state cannot be maintained.

(2) The source of power inspection UAV charging, for the sake of convenience, it is best to come directly from the transmission line. Since the wireless charging position is near the high voltage transmission line, the influence of strong electromagnetic interference generated by the power line on the charging efficiency must be considered.

(3) However, the voltage on the transmission line is too high to be directly applied, so the stable step-down design of the charging platform must also be considered. In addition, because the charging platform is exposed to nature for a long time, how to protect it is also a problem.

(4) At present, there is no systematic analysis of the design of the magnetically coupled resonant charging method. The relationship between the changes of various parameters and the resonant frequency is not clearly explained. A comprehensive analysis scheme is needed to deal with this type of design.

(5) The power inspection drone needs to check multiple target points in one voyage. In order to make the drone save power as much as possible, it is necessary to make a reasonable path planning for the target point to be inspected.

\section{Solutions}

For the time being, some studies have proposed some ways and methods to solve the above problems. 
(1) For the problem 1, the literature [11] gives a constant frequency control strategy based on the dynamic tuning of the phase-controlled inductor, which can basically maintain the resonance state under low power. However, since this is a control strategy based on the floating frequency method, there will still be small floating changes during operation. When the operating frequency of the system is high, the loss caused by the floating is still relatively large. Therefore, the literature proposes a method of fixing the operating frequency to the rated frequency and solving the problem by dynamic tuning, that is, turning the problem into the resonance state of the detection system.

(2) For question 2, the problem to be considered is to achieve effective electromagnetic shielding. In [12], the three-dimensional electric field of the ground with shielded lines is calculated. By calculating the domain mapping, the magnetic field calculation formula of the finite-width flat shielded area actually used in the engineering is derived, and the shielding scheme can be designed for the specific line.

(3) The current power electronics technology can successfully build a high-power transformer and change the voltage amplitude. The premise is that the components can withstand high voltage, that is, the material and structure of the components are the key to support this research. Or you can take a step-by-step transformation to reduce the voltage intensity step by step. The protection of the charging platform is also a research problem of surface materials, which requires better material support to complete the design.

(4) Such a comprehensive analysis program requires a large amount of research data and methods to determine. At present, there is no further research on the wireless charging method of electric power inspection UAVs. Therefore, with the passage of time and deep research, this comprehensive analysis plan will be gradually formed and improved.

(5) The literature [13] designed a polar coordinate coding method to solve the limitation of the maximum path deflection angle, the minimum step size and the maximum number of path nodes. The genetic algorithm is used to solve the UAV line corridor inspection path. During the inspection, a reasonable path was designed and optimized according to the safety distance and path. The experimental simulation proved that the scheme is feasible. New ideas for the overall analysis process for this type of solution are provided.

\section{Conclusion}

With the environment of grid globalization, the expansion of power grid scale is inevitable, so the task of power inspection will become heavier and heavier, and more and more intelligent products such as drones will be required to participate in the inspection, and its magnetic coupling resonance the study of charging methods has a strong practical significance. At present, the application of the charging technology on the UAV still faces various problems, but with the continuous development of science and technology, these problems will eventually be solved in the future, and artificial intelligence will greatly reduce the workload of the power industry such as inspection.

\section{References}

1. Fan Xingming, Mo Xiaoyong, Zhang Xin. Research Status and Application of Magnetically Coupled Resonant Radio Energy Transmission. Transactions of China Electrotechnical Society, 28 (12): 75$82+99$ (2013).

2. Aristeidis Karalis, J.D. Joannopoulos, Marin Soljacic. Efficient wireless non-radiative mid-range energy transfer. Annals of physics, 323 (I): 34-38 (2008).

3. T.Imura, H.Okabe, Y.Hori, Basic experimental study on helical antennas of wireless power transfer for Electric Vehicles by using magnetic resonant couplings.

4. Teck CB, Imura T, Kato M, et al. Basic study of improving efficiency of wireless power transfer via magnetic resonance coupling based on impedance matching. IEEE International Symposium on Industrial Electronics. Bar Italy: IEEE, (2010).

5. Seung-Hwan L, Lorenz R D. Development and validation of model for 95\%-efficiency $220-\mathrm{W}$ wireless power transfer over a $30-\mathrm{cm}$ air gap $[\mathrm{J}]$. IEEE Trans. on Industry Applications, 47 (6): 2495 2504 (2011).

6. Zhang Xiaozhuang. Magnetic coupling resonant wireless energy transmission distance characteristics and its experimental device. Harbin: Harbin Institute of Technology (2009).

7. Fu Wenzhen, Zhang Bo, Qiu Dongyuan. Research on frequency tracking resonant coupled power wireless transmission system. Frequency Converter World, 08: 41-46 (2009).

8. Huang Hui, Huang Xueliang, Tan Linlin. Research on wireless power transmission and reception device based on magnetic field resonance coupling, New Technology of Electrical Engineering and Energy, 30 (1): 32-35 (2011).

9. Geng Yuan, Sun Yue, Dai Xin, Su Yugang, Wang Zhihui. Modeling and Analysis of Magnetic Energy Mode Radio Energy Transmission System. Proceedings of the CSEE, 32 (12): 155-160 (2012).

10. Zhang Xian, Yang Qingxin, Chen Haiyan, Li Yang, Zhang Xin, Jin Liang. Study on Frequency Splitting Characteristics of Electromagnetic Coupling Resonance Energy Transfer System. Proceedings of the CSEE, 32 (09): 167-173 +24 (2012).

11. Zhao Wei. Research on wireless charging technology of drone hover based on radio energy transmission mode. Chongqing University, (2015).

12. Xiao Dongping. Research on $3 \mathrm{D}$ calculation model and shielding measures of electromagnetic field of UHV AC transmission line. Chongqing University (2009).

13. Xiong Dian. Research and application of patrol path planning for transmission line UAV. Wuhan University of Science and Technology (2014). 